\title{
Six-Minute Walking Test (6MWT) Results Assessment in Pulmonary Sarcoidosis Patients
}

\section{Katayoon Samadi ${ }^{*}$, Atefeh Abedini ${ }^{2}$, Shahram Kharabian ${ }^{1}$ and Lida Rezaian ${ }^{1}$}

${ }^{1}$ Chronic Respiratory Diseases Research Center, National Research Institute of Tuberculosis and Lung Diseases (NRITLD), Shahid Beheshti University of Medical Sciences, Tehran, Iran

${ }^{2}$ Tracheal Diseases Research Center, NRITLD, Shahid Beheshti University of Medical Sciences, Tehran, Iran

\begin{abstract}
Background and objectives: Sarcoidosis is an inflammatory disease that affects multiple organs including the muscles which may lead to physical intolerance. The 6 minute walking test (6MWT) is a method that is frequently used to assess physical capacity in these patients. The aim of this study is to investigate 6MWT results in sarcoidosis patients with different radiographic stages, medication therapy, and spirometric parameters.
\end{abstract}

Materials and methods: We assessed 6MWD and oxygen desaturation as outcome measure of 6MWT in 71 sarcoidosis patients categorized into four groups according to Scadding criteria. We also compared 6MWD among 30 patients based on the medication therapy in three groups 1) prednisolone 2) prednisolone plus methotrexate 3) hydroxychloroquine plus prednisolone). Also, correlation between 6MWD and spirometric parameters results including FEV1, FEV1/FVC were investigated in 24 patients.

Results: There was statically significant correlation between oxygen desaturation and sarcoidosis severity; but, in spite of finding a tendency in more severe sarcoidosis stages to walk shorter distance the correlation between 6MWD and sarcoidosis severity was not significant. In exploring 6MWD among different medication groups it turned out that patients who were taking methotrexate and prednisolone tended to walk shorter distance in comparison to patients were taking prednisolone alone.

Conclusion: Oxygen desaturation during 6MWT is another measure outcome of this test which would be recommended to be considered in assessing sarcoidosis severity. 6MWT can be considered not only to help assess sarcoidosis expansion in body but also help in better medication choose in these patients.

Keywords: Sarcoidosis; 6MWT; Rehabilitation; Spirometric results

\section{Introduction}

Sarcoidosis is an inflammatory disease that affects multiple organs, has undetermined origins, and is associated with non-caseating granulomaepithelioid cells and lymphocytic alveolitis [1,2]. Although pulmonary and constitutional symptoms are prevalent in sarcoidosis patients, $50 \%$ of them are asymptomatic and are only diagnosed unexpectedly after abnormalities are discovered in chest X-rays $[3,4]$. Sarcoidosis affects any organ in the body, including the muscles, which may present with signs and symptoms of arthritis, fatigue, and physical intolerance [5]. Fatigue is a very common complaint in $30 \%$ to $90 \%$ of sarcoidosis patients and is also frequently associated with reduced muscle force and exercise capacity [6-8].

To determine how far the disease has progressed, several methods, including complete and precise physical examination, chest X-ray (CXR), and pulmonary function test (PFT), should be used, and for further evaluation, computed tomography (CT) scanning and an exercise test are recommended. Each method has different sensitivity for different stages of the disease; for example, PFT is more sensitive than CXR to investigate disease expansion in the initial stage of the disease [9]. However, CXR is a widely available modality and, because of its low cost, is frequently used for this purpose and for disease staging.

Since sarcoidosis tends to involve upper pulmonary lobes rather than lower pulmonary lobes, it would be more appropriate to explore pulmonary parenchymal involvement and function during exercise. The 6-minute walking test (6MWT) is a simple, low-cost, reproducible method that is frequently used to assess physical capacity in patients with interstitial lung diseases $[10,11]$. Different studies have shown impairment in sarcoidosis patients based on their 6MWT results, which is indicative of physical intolerance [12-15].

Multiple measures, such as spirometric parameters (forced vital capacity [FVC] and forced expiratory volume in first second [FEV1]) and corticosteroid therapy, have been investigated to evaluate different aspects of 6MWT results as an independent factor.

A few studies have investigated different outcome measures of the $6 \mathrm{MWT}$ in sarcoidosis patients, but to our knowledge our study is the second to consider oxygen $\left(\mathrm{O}_{2}\right)$ desaturation (the difference between $\mathrm{O}_{2}$ saturation $\left[\mathrm{SaO}_{2}\right]$ at the beginning of the test and at peak exercise level) as a separate outcome in relation to sarcoidosis severity.

Moreover, several studies of sarcoidosis patients have assessed corticosteroid or overally systemic therapy based on the results of this test, but our study assessed distance walked in the 6MWT (6MWD) in sarcoidosis patients who received three common medication therapies (prednisolone, methotrexate, hydroxychloroquine). No study of sarcoidosis patients has been conducted in Iran with the same focus.

The aim of this study is to investigate 6MWT results in sarcoidosis patients with different radiographic stages, medication therapy, and spirometric parameters.

\section{Materials and Methods}

This study included 71 patients who were diagnosed with

${ }^{*}$ Corresponding author: Katayoon Samadi, Chronic Respiratory Diseases Research Center, National Research Institute of Tuberculosis and Lung Diseases (NRITLD), Shahid Beheshti University of Medical Sciences, Tehran, Iran, Tel: 00989129349682; E-mail: Kati.samadi@gmail.com

Received April 06, 2016; Accepted May 04, 2016; Published May 07, 2016

Citation: Samadi K, Abedini A, Kharabian S, Rezaian L (2016) Six-Minute Walking Test (6MWT) Results Assessment in Pulmonary Sarcoidosis atients. J Pulm Respir Med 6: 341. doi:10.4172/2161-105X.1000341

Copyright: @ 2016 Samadi K, et al. This is an open-access article distributed under the terms of the Creative Commons Attribution License, which permits unrestricted use, distribution, and reproduction in any medium, provided the original author and source are credited. 
pulmonary sarcoidosis and admitted to the outpatient sarcoidosis clinic at Masih Daneshvari Hospital in Tehran, Iran. Patients were diagnosed based on American Thoracic Society/European Respiratory Society and World Association of Sarcoidosis and Other Granulomatous Disorders guidelines [5]. Clinical data, including weight, age, sex, and type of treatment, were recorded from the patients' files. Patients were divided into two groups chronic sarcoidosis and acute sarcoidosis according to their clinical records, process and length of disease, and disease symptoms as assessed by their pulmonologist. The results of the patients' CXRs, which were taken on their last visit day at clinic, were examined by their pulmonologist. The patients were then categorized into four groups based on Scadding criteria [5], which started with stage 1 , defined as hilar lymphadenopathy without parenchymal changes, and ended with stage 4 , defined as highest involvement that presents with parenchymal fibrosis. Because all patients had lung involvement, we ignored stage 0 .

Since the patients' last PFT results were recorded retrospectively and because of an inability to obtain all patients' clinical records, we were able to note the PFT results of only 24 patients. However, all tests were performed in the Masih Daneshvari Hospital spirometry center according to American Thoracic Society guidelines [16]. FVC and FEV1 and FEV1/FVC were measured by pneumotachograph (Masterlab, Jaeger, Wurzburg, Germany) and expressed as percentage of predicted value. All PFTs were conducted 1 month before the patients' $6 \mathrm{MWT}$

All 71 patients took standard 6MWTs using American Thoracic Society guidelines [17]. The tests were conducted along a 30-meter flat, straight corridor, and $\mathrm{SaO}_{2}$ was recorded automatically and continuously every 30 seconds. Each patient relaxed for at least 10 minutes before the $6 \mathrm{MWT}$ commenced. The patients' heart rate, blood pressure, and $\mathrm{SaO}_{2}$ were measured at the beginning of the test. If respirational or muscular tiredness occurred during the $6 \mathrm{MWT}$, walking speed was reduced to a bearable level and test was stopped if participants felt chest pain or experienced severe dyspnea or muscle spasms, or if patients asked to stop because of their inability to continue.

In case of chest pain, minor dyspnea, or sweating, patients were allowed to take a break and then restart the test. At the end, patients' heart rate, blood pressure, $\mathrm{SaO}_{2}$, and the distance walked during 6 minutes were measured. $\mathrm{SaO}_{2}$ was considered the gradient between the saturation measured at the beginning of the test and the lowest saturation measured during the test.

Because of an inability to obtain all patients' clinical records (as with the patients' PFT results), we were able to obtain the medical management profiles of only 30 patients. These 30 patients were categorized into three groups according to the medications they were taking for management of their sarcoidosis:

1) Patients who were taking prednisolone.

2) Patients who were taking prednisolone and methotrexate.

3) Patients who were taking prednisolone and hydroxychloroquine.

Informed and written consent were obtained from all 71 patients. Patients' confidentiality was maintained.

Patients were excluded if they had coincidental chronic lung disease or acute pulmonary infection, or if they were dependent on consistent supplemental oxygen therapy (to eliminate the bias that may affect desaturation measurement during the 6MWT).

\section{Statistical analysis}

Disease chronicity, sarcoidosis severity score, distance walked in
$6 \mathrm{MWT}$, and $\mathrm{SaO}_{2}$ (before and after the $6 \mathrm{MWT}$ ) were measured for all 71 patients. Also, spirometry indicators were recorded for 24 patients, and the type of treatment regimen was available for 30 patients. Collected data were assessed using SPSS software, version 16 .

To evaluate normality assumption, we used the Shapiro-Wilk normality test. Pearson and Spearman correlation tests were used to assess the relationship between 6MWD or sarcoidosis severity stage with spirometry indicators and $\mathrm{O}_{2}$ desaturation. To adjust for the effect of sex on these relationships, we applied partial correlation tests. Furthermore, to evaluate the effect of treatment regimen on the distance patients walked in the 6MWT, we used analysis of variance (ANOVA) as well as Tukey post-hoc tests.

\section{Results}

We studied data from $30(42 \%)$ men and 41 (58\%) women. The mean age of the women (49.6 years) was slightly higher than that of the men ( 43.8 years). Although $\mathrm{SaO}_{2}$ measurements for the men and women were roughly equal before the $6 \mathrm{MWT}$, women showed higher $\mathrm{O}_{2}$ desaturation (5.5 liter) than men (3.3 liter). On the other hand, predicted spirometry indicators did not show considerable difference between men and women. Also, normal distribution was recorded for all variables except age, $\mathrm{SaO}_{2}$ after $6 \mathrm{MWT}$, and oxygen desaturation (Table 1).

Correlation test results are presented in Table 2. These results show the relationship between distance walked in the 6MWT and spirometry indicators, (using the Pearson correlation test) and the relationship between sarcoidosis severity degree and distance or oxygen desaturation (using the Spearman correlation test).

Adjusted (by age and sex) correlation coefficients did not differ from unadjusted results, and in both cases, only the correlation between sarcoidosis severity degree and oxygen desaturation was statistically significant (adjusted Spearman's rho $=0.372 ; P=0.003$ ). Figure 1 also shows the average oxygen desaturation by disease severity score for both sexes. As shown in both Table 2 and Figure 1, oxygen desaturation tended to get higher as the disease got more severe.

For the 30 patients for whom treatment information was available, 12 were receiving prednisolone, 13 were receiving prednisolone plus methotrexate, and five were receiving prednisolone plus hydroxychloroquine. Considering sex, age, and disease chronicity, we used a univariate ANOVA test to evaluate the effect of treatment regimen on the distance patients walked in the 6MWT. Table 3 shows the univariate ANOVA results, which indicate that treatment regimen had a significant effect on distance walked by patients in the $6 \mathrm{MWT}$ $(\mathrm{P}=0.007)$. To evaluate the performance of each treatment regimen group, we used Tukey post-hoc analysis, the results of which are presented in Table 4 . These results show that patients who received only prednisolone walked significantly further (average, 112.5 meters) than those who received methotrexate plus prednisone $(\mathrm{P}=0.003)$. However, the distance walked in the 6MWT by those patients who received prednisolone plus hydroxychloroquine was not significantly different than the distance walked by patients in the other two groups.

\section{Discussion}

We performed a cross-sectional study on unselected sarcoidosis patients who were admitted to the outpatient sarcoidosis clinic at Masih Daneshvari Hospital. We investigated the 6MWT results of 71 sarcoidosis patients who were categorized based on the Scadding severity scoring system. The results showed that patients with more severe sarcoidosis tended to have a greater decline in oxygen desaturation during the 
Citation: Samadi K, Abedini A, Kharabian S, Rezaian L (2016) Six-Minute Walking Test (6MWT) Results Assessment in Pulmonary Sarcoidosis atients. J Pulm Respir Med 6: 341. doi:10.4172/2161-105X.1000341

Page 3 of 5

\begin{tabular}{|c|c|c|c|}
\hline \multirow{2}{*}{ Factor } & \multicolumn{3}{|c|}{ Mean \pm SD } \\
\hline & Females (Women) & Males (Men) & Total \\
\hline Age (years) & $49.6 \pm 7.7$ & $43.8 \pm 8.4$ & $47.2 \pm 8.5$ \\
\hline $\mathrm{SpO}_{2}(\%)-$ before $^{*}$ & $93.8 \pm 2.5$ & $93.7 \pm 2.5$ & $93.7 \pm 2.4$ \\
\hline $\mathrm{SpO}_{2}-$ after & $88.6 \pm 5.6$ & $90.7 \pm 4.4$ & $89.5 \pm 5.2$ \\
\hline Distance (meter) (traveled in 6MWT)* & $397.6 \pm 102.8$ & $495.0 \pm 90.0$ & $439.3 \pm 108.3$ \\
\hline FVC - actual ${ }^{*}$ & $2.6 \pm 0.7$ & $3.6 \pm 1.4$ & $2.9 \pm 1.0$ \\
\hline FVC - predicted ${ }^{*}$ & $84.1 \pm 20.3$ & $81.5 \pm 19.5$ & $83.2 \pm 19.6$ \\
\hline $\mathrm{FEV}_{1}-$ actual $^{*}$ & $2.4 \pm 0.7$ & $3.1 \pm 1.3$ & $2.6 \pm 0.9$ \\
\hline $\mathrm{FEV}_{1}$ - predicted $^{*}$ & $84.1 \pm 16.6$ & $79.5 \pm 26.6$ & $82.5 \pm 20.0$ \\
\hline $\mathrm{FEV}_{1} / \mathrm{FVC}^{*}$ & $86.8 \pm 12.8$ & $82.7 \pm 20.4$ & $85.4 \pm 15.5$ \\
\hline Oxygen desaturation & $5.2 \pm 5.7$ & $3.3 \pm 4.0$ & $4.4 \pm 5.1$ \\
\hline
\end{tabular}

6MWT:6 min Walking Test; SD: Standard Deviation; FVC: Forced Vital Capacity; FEV1: Forced Expiratory Volume in First Second; SpO ${ }_{2}$ : Oxygen Saturation. Table 1: Study population characteristics.

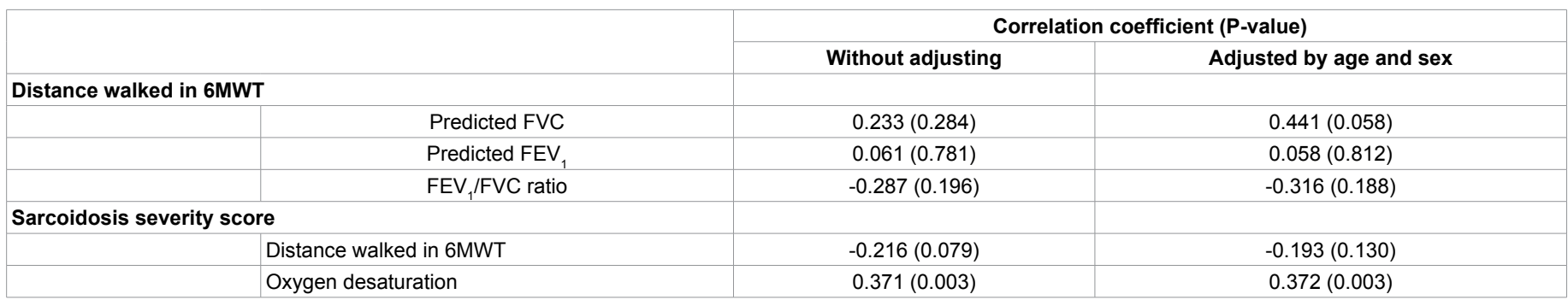

Table 2: Correlation tests with and without adjusting by age and sex.

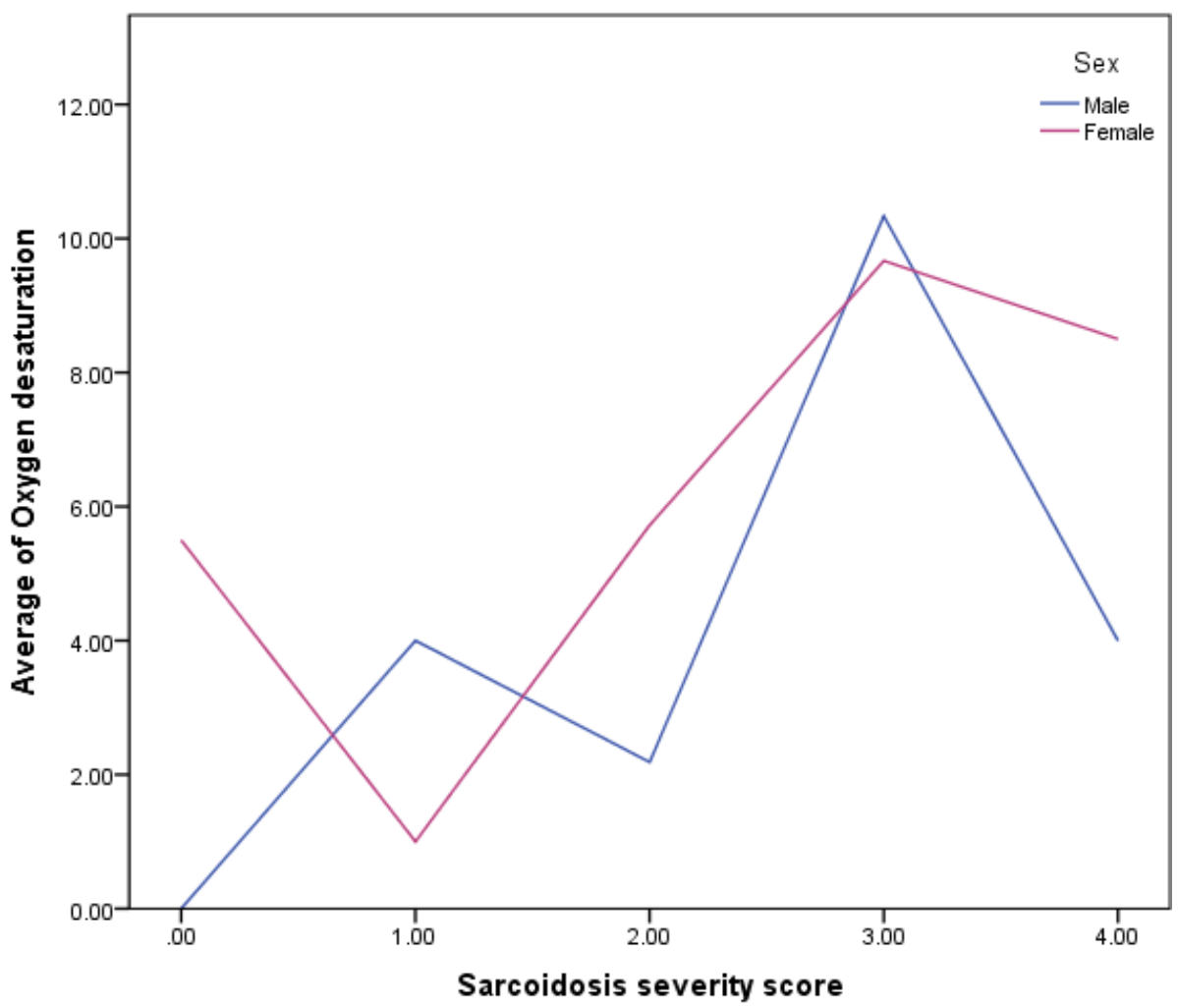

Figure 1: Oxygen desaturation average by sarcoidosis severity score and sex. 


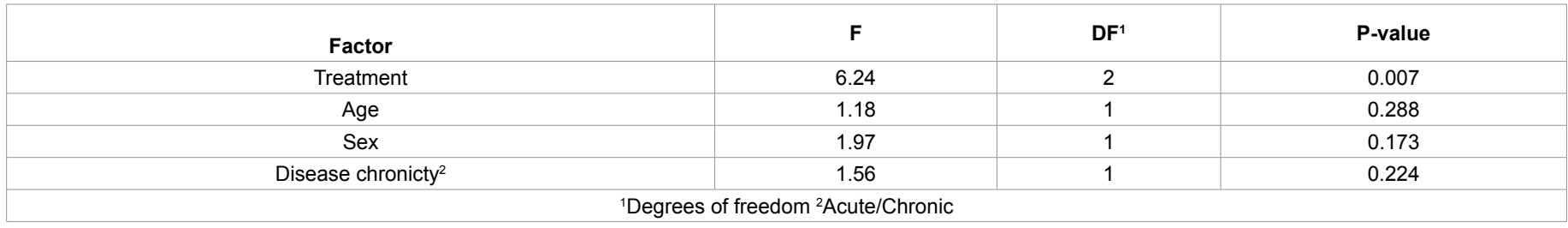

Table 3: Univariate analysis of variance of factors affecting distance walked in 6MWT.

\begin{tabular}{|c|c|c|}
\hline Treatment A & Treatment B & Distance difference (A-B) (95\% Cl) \\
\hline Prednisolone & Prednisone + Methotrexate & $112.5(36.4-188.5)^{\star}$ \\
\hline Prednisolone & Prednisone + hydroxycholorquin & $75.2(-25.9-176.4)$ \\
\hline Prednisolone + Methotrexate & Prednisone +hydroxycholorquin & $-37.2(-137.2-62.8)$ \\
\hline
\end{tabular}

Table 4: Tukey post-hoc analysis of distance difference between treatment groups.

$6 \mathrm{MWT}$ and were able to walk a shorter distance than patients with only minor sarcoidosis although the walking distance measurement was not statistically significant. Patients with different therapy regimens were able to walk different distances during the 6MWT: patients taking methotrexate plus prednisolone walked shorter distances compared to those who were taking only prednisolone. Spirometric indicators showed no significant correlation between 6MWD and spirometric parameters.

Several factors lead to impaired physical capacity in sarcoidosis patients, including corticosteroid use, myositis, pulmonary hypertension, depression, muscle weakness, respirational muscle weakness, increased alpha interleukin level, inactivity and depression [18-22].

The 6MWT has been used to study physical capacity in patients with different pulmonary diseases, such as chronic obstructive pulmonary disease, interstitial pulmonary fibrosis, and pulmonary hypertension [23-25].

A few studies have investigated this test in sarcoidosis patients. In all studies, women tended to walk shorter distances than men $[15,26,27]$, although this is found in healthy people as well $[28,29]$. Spruit et al. [14] found lower 6MWD in fatigued sarcoidosis patients compared to healthy participants. In another study, Kabitz [13] et al. reported reduced $6 \mathrm{MWD}$ in male sarcoidosis patients as compared to healthy participants. Likewise, two other studies reached the same conclusion [12,15]. In another study, Drent et al. [29] found that fatigued sarcoidosis patients may suffer more from physical intolerance than non-fatigued patients.

Baughman et al. [12] studied 6MWD in sarcoidosis patients with different radiographic stages and observed that with higher radiographic grades, patients tended to have lower 6MWD, although this correlation was not statistically significant. These results are similar to those found in our study and in another study by Alhamad [15]. Baughman et al. [12] also compared 6MWD in three groups of patients with different radiographic parenchymal pattern no parenchymal changes (stage 0 and 1), with parenchymal changes (stage 2 and 3 ), and with fibrosis (stage 4 ) and found no difference by this categorization.

In 48 sarcoidosis patients, Medinger et al. [30] studied the correlation between different radiographic stages and other factors such as diffusing capacity of the lungs for carbon monoxide, total lung capacity, and spirometric parameters. They concluded that this correlation is specifically significant in relation to the gradient in arterial oxygen tension between peak exercise during 6MWT and resting measurement, which is similar to our test results, although we considered pulse oximetry for this measure.

In a univariate analysis model, Baughman et al. [12] identified lowest oxygen saturation and FVC as independent variables affecting 6MWD. Also, they identified a positive correlation between 6MWD and FEV1, which is in contrast to the results of Alhamad et al. [27], who found that FEV1 was an independent variable affecting 6MWD. Moreover, Alhamad et al. [27] did not find any statistically significant correlation between $6 \mathrm{MWD}$ and lowest oxygen saturation, in contrast to the results found by Baughman et al. Despite finding a positive correlation between spirometric parameters (FVC and FEV1) and $6 \mathrm{MWD}$ in our study, this relation was not statistically significant, which is probably due to our small sample size in comparison to the sample sizes in the two aforementioned studies. Alhamad et al. [27] recommended another outcome measure of 6MWT distance saturation product instead of $6 \mathrm{MWD}$, which was shown to be related to many factors affecting $6 \mathrm{MWT}$ performance that $6 \mathrm{MWD}$ is not able to recognize alone, like systemic therapy, pulmonary hypertension, and CT fibrosis.

Although, previous works showed that spirometric indicators were independent factors influencing $6 \mathrm{MWD}$, but 6MWD might be impaired even in patients with completely normal PFT [31].

Several previous investigators studied the effect of systemic medication on 6MWD results; all of them drew a similar conclusion, namely that systemic therapy, specifically corticosteroid therapy, results in a reduction in 6MWD [14,26,27]. In a study of 26 sarcoidosis patients conducted by Alhamad [15], they investigated 6MWD and divided the patients into four groups: 1) those receiving no treatment; 2) those taking prednisolone; 3) those taking prednisolone and azathioprine; and 4) those taking prednisolone and methotrexate. They found that patients taking prednisolone alone tended to walk shorter distances than patients in the other three groups but not to a level of statistical significance. On the other hand, in our study of 30 patients, those who were taking methotrexate with prednisolone walked shorter distances compared to those who were taking prednisolone alone. This result is probably affected by duration of therapy and dose of medication, which was not considered in either study. To evaluate the effect of treatment regimen on the distance patients could walk on the 6MWT, we considered the effect of patients' sex, age, and disease chronicity. Although sex was a significant factor affecting distance walked using simple $\mathrm{t}$-test analysis $(\mathrm{t}=4.137 ; \mathrm{df}=68 ; P<0.001)$, its effect was not significant when we evaluated it using the ANOVA model along with other factors. The small amount of available treatment regimen data led the effect of sex on distance to be captured by other factors and make sex insignificant. Although it may be a limitation of our study, we still 
evaluated sex using the ANOVA model to determine the adjusted effect of treatment on distance (without assessing the effect of sex itself).

One known limitation of our study is that many data were obtained only retrospectively from clinical records. Also, Masih Daneshvari Hospital is a tertiary care center, which may cause selection bias. Another limitation of our study is the small sample size for spirometric and therapy evaluation.

\section{Conclusion}

Oxygen desaturation as another 6MWT outcome measure in relation with sarcoidosis severity. Sarcoidosis patients receiving methotrexate therapy exhibited a greater decline in $6 \mathrm{MWD}$ in comparison to patients taking prednisolone alone. Considering this effect on physical capability can help physicians choose more appropriate therapy based on their patients' physical tolerance.

\section{Acknowledgment}

We thank John H. McCool, MA, of Houston, Texas, USA, for providing editorial assistance in the writing of this article.

\section{References}

1. Hunninghake GW, Costabel U, Ando M, Baughman R, Cordier JF, et al. (1999) ATS/ERS/WASOG statement on sarcoidosis. American Thoracic Society/ European Respiratory Society/World Association of Sarcoidosis and other Granulomatous Disorders. Sarcoidosis Vasc Diffuse Lung Dis 16: 149-173.

2. Müller-Quernheim J (1998) Sarcoidosis: immunopathogenetic concepts and their clinical application. Eur Respir J 12: 716-738.

3. Lynch JP, Kazerooni EA, Gay SE (1997) Pulmonary sarcoidosis. Clin Chest Med 18: 755-785.

4. Baughman RP, Teirstein AS, Judson MA, Rossman MD, Yeager H, et al. (2001) Clinical characteristics of patients in a case control study of sarcoidosis. Am J Respir Crit Care Med 164: 1885-1889.

5. [No authors listed] (1999) Statement on sarcoidosis. Joint Statement of the American Thoracic Society (ATS), the European Respiratory Society (ERS) and the World Association of Sarcoidosis and Other Granulomatous Disorders (WASOG) adopted by the ATS Board of Directors and by the ERS Executive Committee, February 1999. Am J Respir Crit Care Med 160: 736-755.

6. Wirnsberger RM, de Vries J, Wouters EF, Drent M (1998) Clinical presentation of sarcoidosis in The Netherlands an epidemiological study. Neth J Med 53: 53-60.

7. De Vries J, Michielsen H, Van Heck GL, Drent M (2004) Measuring fatigue in sarcoidosis: the Fatigue Assessment Scale (FAS). Br J Health Psychol 9: 279-291.

8. Kumor K, Pierzcha ÅK (2006) [The problem of fatigue in neurological disorders]. Wiad Lek 59: 685-691.

9. Miller A, Teirstein AS, Chuang MT (1977) The sequence of physiologic changes in pulmonary sarcoidosis: correlation with radiographic stages and response to therapy. Mt Sinai J Med 44: 852-865.

10. Bourbonnais JM, Samavati L (2008) Clinical predictors of pulmonary hypertension in sarcoidosis. Eur Respir J 32: 296-302.

11. Flaherty KR, Andrei AC, Murray S, Fraley C, Colby TV, et al. (2006) Idiopathic pulmonary fibrosis: prognostic value of changes in physiology and six-minutewalk test. Am J Respir Crit Care Med 174: 803-809.

12. Baughman RP, Sparkman BK, Lower EE (2007) Six-minute walk test and health status assessment in sarcoidosis. Chest 132: 207-213.

13. Kabitz HJ, Lang F, Walterspacher S, Sorichter S, Müller-Quernheim J, et al (2006) Impact of impaired inspiratory muscle strength on dyspnea and walking capacity in sarcoidosis. Chest 130: 1496-1502.
4. Spruit MA, Thomeer MJ, Gosselink R, Troosters T, Kasran A, et al. (2005) Skeletal muscle weakness in patients with sarcoidosis and its relationship with exercise intolerance and reduced health status. Thorax 60: 32-38.

15. Alhamad EH (2009) The six-minute walk test in patients with pulmonary sarcoidosis. Ann Thorac Med 4: 60-64.

16. [No authors listed] (1995) Standardization of Spirometry, 1994 Update. American Thoracic Society. Am J Respir Crit Care Med 152: 1107-1136.

17. ATS Committee on Proficiency Standards for Clinical Pulmonary Function Laboratories (2002) ATS statement: guidelines for the six-minute walk test. Am J Respir Crit Care Med 166: 111-117.

18. Silverstein A, Siltzbach LE (1969) Muscle involvement in sarcoidosis. Asymptomatic, myositis and myopathy. Arch Neurol 21: 235-241.

19. Baughman RP, Sparkman BK, Lower EE (2007) Six-minute walk test and health status assessment in sarcoidosis. Chest 132: 207-213.

20. Al-Nozha MM, Al-Hazzaa HM, Arafah MR, Al-Khadra A, Al-Mazrou YY, et al. (2007) Prevalence of physical activity and inactivity among Saudis aged 3070 years. A population-based cross-sectional study. Saudi Med J 28: 559-568.

21. Baydur A, Alsalek M, Louie SG, Sharma OP (2001) Respiratory muscle strength, lung function, and dyspnea in patients with sarcoidosis. Chest 120 102-108.

22. Chang B, Steimel J, Moller DR, Baughman RP, Judson MA, et al. (2001) Depression in sarcoidosis. Am J Respir Crit Care Med 163: 329-334.

23. Chetta A, Aiello M, Foresi A, Marangio E, D'lppolito R, et al. (2001) Relationship between outcome measures of six-minute walk test and baseline lung function in patients with interstitial lung disease. Sarcoid Vasc Diffuse Lung Dis 18: 170175.

24. Lama VN, Flaherty KR, Toews GB, Colby TV, Travis WD, et al. (2003) Prognostic value of desaturation during a 6-minute walk test in idiopathic interstitial pneumonia. Am J Respir Crit Care Med 168: 1084-1090.

25. Eaton T, Young P, Milne D, Wells AU (2005) Six-minute walk, maximal exercise tests: reproducibility in fibrotic interstitial pneumonia. Am J Respir Crit Care Med 171: 1150-1157.

26. Baughman RP, Sparkman BK, Lower EE (2007) Six-minute walk test and health status assessment in sarcoidosis. Chest 132: 207-213.

27. Alhamad EH, Shaik SA, Idrees MM, Alanezi MO, Isnani AC (2010) Outcome measures of the 6 minute walk test: relationships with physiologic and computed tomography findings in patients with sarcoidosis. BMC Pulm Med 10: 42-45.

28. Enright PL, Sherrill DL (1998) Reference equations for the six-minute walk in healthy adults. Am J Respir Crit Care Med 158: 1384-1387

29. Drent M, Wirnsberger RM, de Vries J, van Dieijen-Visser MP, Wouters EF, et al. (1999) Association of fatigue with an acute phase response in sarcoidosis. Eur Respir J 13: 718-722.

30. Medinger AE, Khouri S, Rohatgi PK (2001) Sarcoidosis: the value of exercise testing. Chest 120: 93-101.

31. Delobbe A, Perrault H, Maitre J, Robin S, Hossein-Foucher C, et al. (2002) Impaired exercise response in sarcoid patients with normal pulmonary function Sarcoid Vasc Diffuse Lung Dis 19: 148-153. 